\title{
A 92 Days Run of Venovenous Extracorporeal Membrane Oxygenation
}

\author{
Manoj Kumar Sahu ${ }^{1}$ Chalattil Bipin ${ }^{1}$ Sourabh Pahuja ${ }^{2}$ Sarvesh Pal Singh ${ }^{1}$ Vijay Hadda ${ }^{2}$
}

\author{
${ }^{1}$ Department of Cardiothoracic and Vascular Surgery, CN Centre, All \\ India Institute of Medical Sciences, Ansari Nagar, New Delhi, India \\ ${ }^{2}$ Department of Pulmonary Medicine, All India Institute of Medical \\ Sciences, New Delhi, India
}

Address for correspondence Manoj K Sahu, MD, DNB, Department of Cardiothoracic and Vascular Surgery, All India Institute of Medical Sciences, CTVS office, 7th floor, CN Centre, New Delhi, 110029, India (e-mail: drmanojsahu@gmail.com).

J Card Crit Care:2020;4:147-152

\begin{abstract}
Keywords

- H1N1 influenza

- acute respiratory distress syndrome

- venovenous extracorporeal membrane oxygenation

Background Venovenous extracorporeal membrane oxygenation (VV-ECMO) is an established life-saving procedure for severe acute respiratory failure due to various causes. In general, the duration of ECMO ranges from 1 to 2 weeks, with withdrawal recommended if no improvement is noted. We report a case of respiratory failure due to acute respiratory distress syndrome (ARDS) following influenza A infection, supported with a long ECMO run and the lessons learned from this experience.

Case Report A 40-year-old female weighing $120 \mathrm{~kg}$ with pneumonia following H1N1 influenza was transferred to our hospital on mechanical ventilation for worsening respiratory distress. On admission, she presented with bilateral diffuse infiltrates on chest $\mathrm{X}$-ray and severe hypoxemia with a partial pressure of oxygen in arterial blood/fraction of inspired oxygen concentration ratio $\left(\mathrm{PaO}_{2} / \mathrm{FiO}_{2}\right)$ of 85 at $\mathrm{FiO}_{2}$ of 0.8 on endotracheal intubation and mechanical ventilation. Acinetobacter Baumannii was isolated from respiratory secretions; antibiotics were revised as per sensitivity. Her respiratory status further deteriorated over next 96 hours in spite of maximally optimized mechanical ventilation. VV-ECMO was established on 4th day of mechanical ventilation in our hospital. Thereafter, she underwent a prolonged ECMO run with respiratory improvement starting to show some promise only by 86th day of ECMO. Weaning process was initiated gradually. However, on 88th day of ECMO the patient had an episode of seizure followed by low Glasgow Coma Scale (GCS) score (3T/15). The patient did not recover from the cerebral insult and based on clinical neurological examination including apnea test, brain death was determined within next 48 hours and the ECMO was called off.

Conclusions Prolonged ECMO therapy poses many challenges and might be considered if the primary cause of respiratory failure necessitating ECMO is expected to resolve or a feasibility of lung transplantation is contemplated. Good team dynamics and appropriate counselling to the family are of utmost importance managing the patients on prolonged ECMO.
\end{abstract}

published online October 8, 2020
DOI https://doi.org/

10.1055/s-0040-1716637

ISSN 2457-0206. (c) 2020. Official Publication of The Simulation Society (TSS), accredited by International Society of Cardiovascular Ultrasound (ISCU).

This is an open access article published by Thieme under the terms of the Creative Commons Attribution-NonDerivative-NonCommercial-License, permitting copying and reproduction so long as the original work is given appropriate credit. Contents may not be used for commercial purposes, or adapted, remixed, transformed or built upon. (https://creativecommons.org/licenses/by-nc-nd/4.0/)

Thieme Medical and Scientific Publishers Pvt. Ltd., A-12, 2nd Floor, Sector 2, Noida-201301 UP, India 


\section{Introduction}

Extracorporeal membrane oxygenation (ECMO) therapy has shown promising results for patients with severe respiratory failure unresponsive to conventional mechanical ventilation. The Extracorporeal Life Support Organization (ELSO) guidelines suggest a treatment period of 2 weeks and withdrawal if there is no improvement. ${ }^{1}$ This time frame is not standard/ fixed and may vary according to the primary cause of respiratory failure, the function of other organ systems, and probability of recovery or chances of lung transplantation with prolonged ECMO application. ${ }^{1-3}$ We report a case of severe acute respiratory distress syndrome (ARDS) caused by H1N1 influenza with superadded bacterial infection, the patient had a long ECMO run of 92 days and developed convulsions followed by coma on 88th day just when she was showing signs of improvement. We lost our patient from a probable major intracerebral bleed at the end but we faced many challenges during this longest venovenous extracorporeal membrane oxygenation (VV-ECMO) run in our hospital.

\section{Case Report}

A 40-year-female patient weighing $120 \mathrm{~kg}$ was tested positive for H1N1influenza by reverse transcriptase polymerase chain reaction and was treated for superadded bacterial pneumonia in a local hospital with broad spectrum antimicrobials including piperacillin-tazobactam, amikacin, voriconazole, and oseltamivir. Her respiratory condition deteriorated and she was put on invasive mechanical ventilation and transferred to our hospital for further management.

On admission to our intensive care unit, her oxygen saturation was $85 \%$ with controlled mechanical ventilation with $450 \mathrm{~mL}$ tidal volume, $30 / \mathrm{min}$ respiratory rate, $12 \mathrm{cmH}_{2} \mathrm{O}$ positive end-expiratory pressure (PEEP), and $80 \%$ fraction of inspired oxygen $\left(\mathrm{FiO}_{2}\right)$. She was running high fever at $103.5^{\circ} \mathrm{F}$, tachycardic(160/minute), her blood pressure was 136/69 mm $\mathrm{Hg}$, and urine output was satisfactory. Auscultation revealed fine crackles in all lung fields. Laboratory blood investigations were unremarkable except for renal impairment (urea 96mg/ $\mathrm{dL}$ and creatinine $1.7 \mathrm{mg} / \mathrm{dL}$ ), and raised C-reactive proteins $(80 \mathrm{mg} / \mathrm{dL}$ ). Chest X-ray showed bilateral diffuse infiltrates on all four quadrants ( - Fig. 1). Arterial blood gas analysis showed low partial pressure of oxygen $\left(\mathrm{PaO}_{2}\right)$ with a partial pressure of oxygen in arterial blood/fraction of inspired oxygen concentration $\left(\mathrm{PaO}_{2} / \mathrm{FiO}_{2}\right)$ ratio of 85 . Her renal functions reverted to normal and she became afebrile 48 hours after antibiotic escalation to cefoperazone-sulbactam, levofloxacin, teicoplanin (after sending all cultures), and fluid management. Subsequently, blood culture yielded Acinetobacter and colistin was added. Her respiratory parameters continued to deteriorate by the 4 th admission day to a $\mathrm{PaO}_{2} / \mathrm{FiO}_{2}<80$, with $15 \mathrm{cmH}_{2} \mathrm{O}$ PEEP, $100 \% \mathrm{FiO}_{2}$, and lung recruitment maneuvers. Proning was not possible because of morbid obesity. In view of worsening ARDS at a Murray score of 3, VV-ECMO was established after obtaining informed consent. After systemic heparinization with 100 units $/ \mathrm{kg}$, a 19-French return cannula was placed in his right internal jugular vein with the

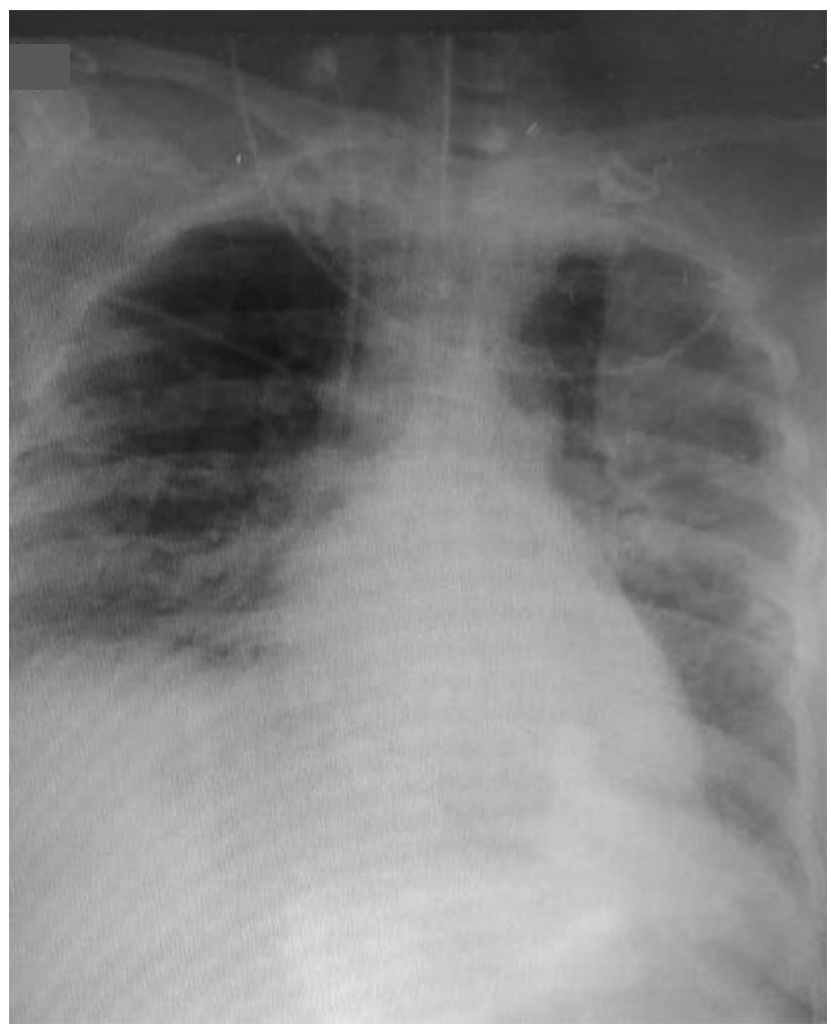

Fig. 1 Chest X-ray on admission to our intensive care unit showing homogeneous opacity in almost whole of left lung and middle and lower lobes of right lung.

distal tip in the right atrium and a 25-French drainage cannula was placed in her right femoral vein with the distal tip in the inferior vena cava at thoracic level 10. Both the cannulae (Bio-Medicus, Medtronic, Minneapolis, Minnesota, United States) were inserted percutaneously and subsequently their position was confirmed by transthoracic echocardiography.

VV-ECMO was initiated at a flow of $5.5 \mathrm{~L} /$ minute with $80 \%$ $\mathrm{FiO}_{2}$ and sweep gas flow of $5 \mathrm{~L}$. Heparin infusion was started at 10 units $/ \mathrm{kg} /$ hour and titrated by activated clotting time monitoring every 2 hours with 180 -second target. The patient had many episodes of intrapulmonary hemorrhage in the initial days of ECMO and she required blood and blood product transfusions from time to time. However, she remained hemodynamically stable, on sedation with fentanyl and midazolam infusions and minimal ventilation. The ECMO ran smoothly at 5 to $6 \mathrm{~L} / \mathrm{min}$ flow at revolution per minute (RPM) of 3,300 throughout the course. Her pulmonary functions did not improve till 10th ECMO day and she required same flows to maintain saturation of 85 to $90 \%$. In view of difficult weaning and for better pulmonary toileting, tracheostomy was done on 12th ECMO day. Subsequently, the patient was awakened, ventilated with intermittent mandatory ventilation and pressure support; her nutrition was maintained through nasogastric tube feeding and continued successfully. The pulmonary parenchyma did not show signs of improvement (radiologically and blood gas wise) in subsequent days and weeks. It seemed as if the last stage of ARDS has attained and fibrotic lung won't improve further (-Figs. 2-4 showing chest X-rays on days 9, 33, and 40). But with progressive 


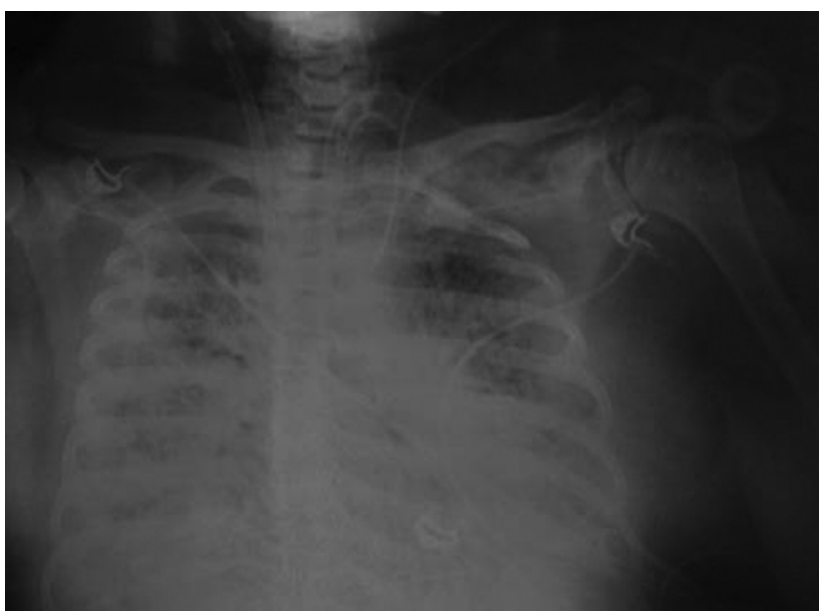

Fig. 2 Chest X-ray on Day 8 of intensive care unit admission-extracorporeal membrane oxygenation instituted.

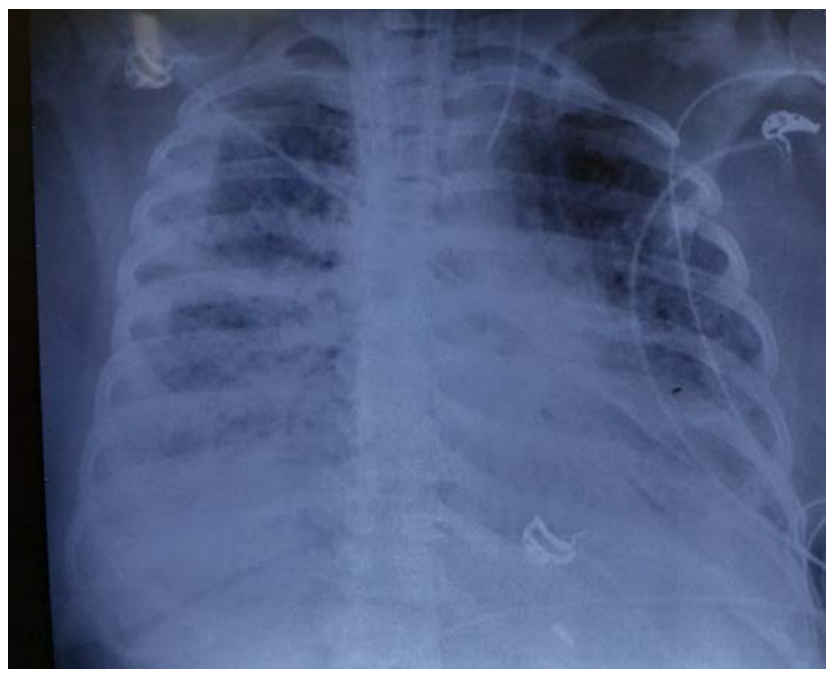

Fig. 3 Chest X-ray on Day 33 extracorporeal membrane oxygenation-looking as if very little improvement in upper lobe of left lung parenchyma.

physiotherapy, pulmonary toileting, and enteral feeding, her cardiovascular system, central nervous system, kidney, and liver functions were maintained at steady state most of the time during the long ECMO run. Though her urine output was adequate, she accumulated large amount of tissue fluid because of inflammation-induced capillary leak and imbalanced plasma oncotic and hydrostatic pressures. Prolonged and continuous systemic inflammation because of mechanical ventilation, extracorporeal circulation, and infection tilted the fluid balance to positive side all these days.In the meanwhile, time elapsed and oxygenator continued to perform like a champion well after its recommended duration of 3 to 4 weeks; when it exceeded 60 days of use, the postmembrane $\mathrm{PaO}_{2}$ started falling and it was changed electively on 65 th day. Unfortunately the second oxygenator got thrombosed and the same was replaced on 76th day ( - Fig. $\mathbf{5}$ ).

Antibiotic changes were required from time to time on the clinical basis when sepsis episodes begin or when new

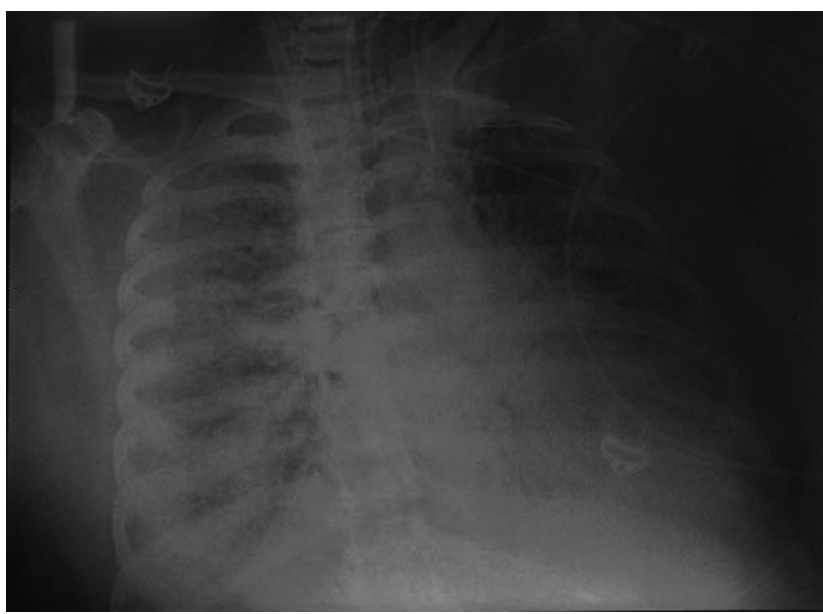

Fig. 4 Chest $X$-ray on Day 40 extracorporeal membrane oxygenation-again deteriorated from the previous little improvement.

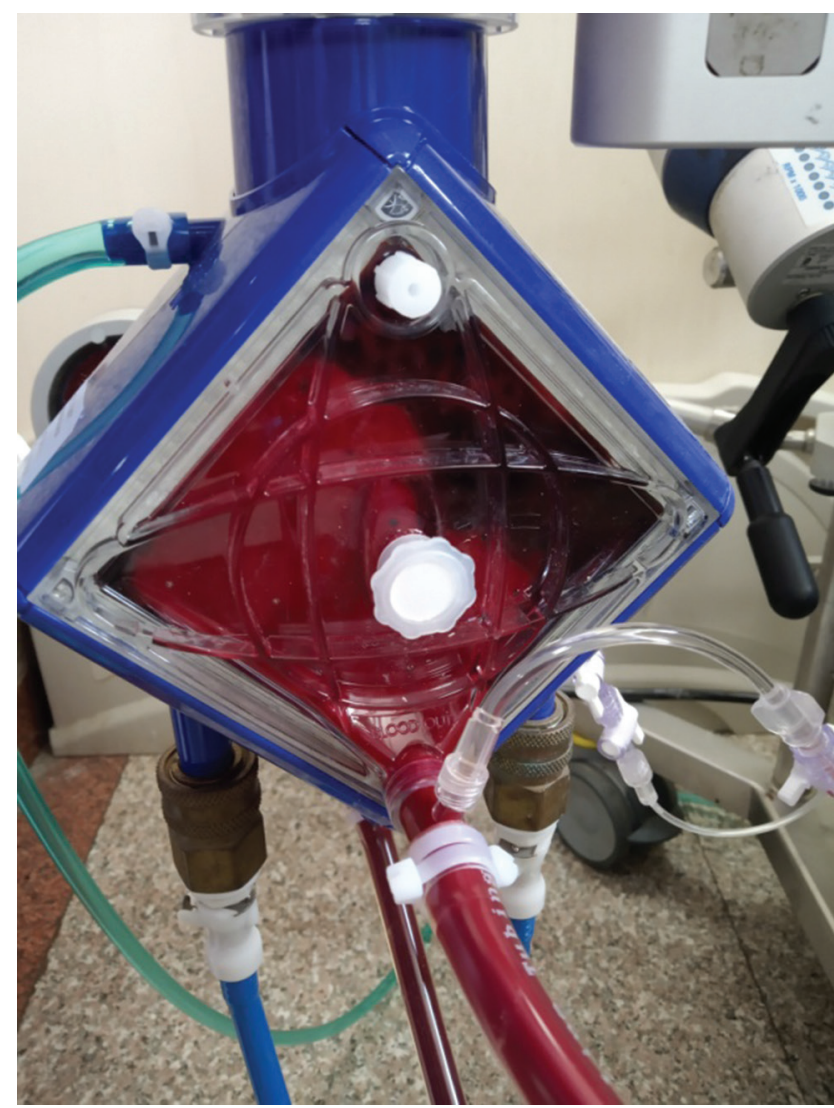

Fig. 5 Thrombus in the second membrane oxygenator on 10th day of change.

pathogens were isolated from cultures and in this process many higher antimicrobials were implemented in cycles. Her hemodynamics and ECMO functions were very steady all through except during the sepsis episodes when she required lower doses of inotropes (dopamine and norepinephrine) and hydrocortisone to support her hemodynamics and renal functions. She also was resuscitated with crystalloids, platelets, plasma, and packed red blood cell transfusions during these sepsis episodes to maintain her tissue perfusion and 
correct her hematocrit and coagulation abnormalities. Her renal functions also got deranged during these crisis times, but the kidneys always recovered with treatment of sepsis.

With aggressive physiotherapy, regular enteral feeds, and counselling, she showed confidence and hope to recover from the probable fibrotic lung changes. But repeated attempts to decrease the pressure support failed and pulmonary functions did not improve. On 60th ECMO day, again respiratory secretion grew Acinetobacter and was treated. Over this period (from 60th till 80th day on ECMO), we faced several hurdles such as frequent dyselectrolytemia, acute kidney injury, hypoalbuminemia, upper gastrointestinal bleeding, hematuria, and feeding intolerance that were managed conservatively.

With prolonged mechanical ventilation, persistence of pulmonary infection and multiple sepsis episodes, confinement to bed (dual cannula VV-ECMO), high body mass index, and interstitial tissue fluid accumulation, she was never considered a candidate for lung transplantation and her only hope was to recover from the primary disease. Hence, when she crossed 80th ECMO day without showing signs of expected pulmonary improvement, our team lost hope and started counselling the family about discontinuation of ECMO therapy.

While discontinuation of ECMO therapy was discussed among caregivers and family, her $\mathrm{PaO}_{2} / \mathrm{FiO}_{2}$ improved to 85 and she showed clinical and radiological improvement on 86th day ( - Fig. 6). We started to decrease the ECMO flows very slowly as she started breathing better with lesser pressure support (PS), but again it was kind of a temporary improvement for couple of days, which did not sustain to wean the patient from ECMO.

On 90th ECMO day, an episode of sudden generalized convulsions occurred that was followed by lower Glasgow Coma Scale (GCS) scores (3T/15). She was treated with one bolus of intravenous (IV) midazolam and regular IV levetiracetam. The neurology service was consulted for evaluation. The

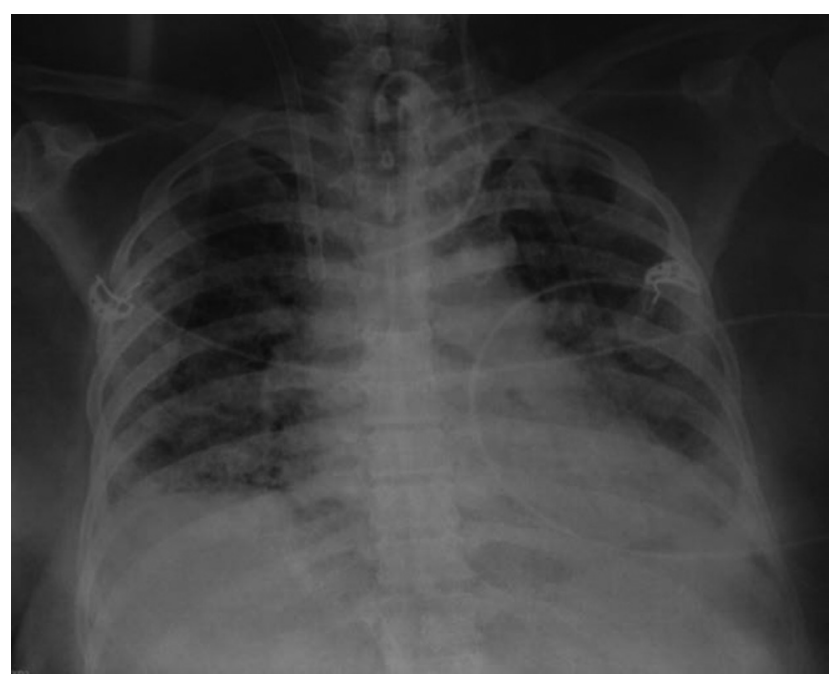

Fig. 6 Chest X-ray on 86th Day of extracorporeal membrane oxygenation showing slow beginning of resolution of acute respiratory distress syndrome. patient did not have repeat seizures in the next 48 hours but did not wake up any more. Computed tomography/magnetic resonance imaging brain could not be performed due to difficulty in moving the sick patient on ECMO to radiology suit. Based on two thorough clinical neurological examinations at 24 hours intervals with findings of irreversible apneic unconsciousness and absent brain stem reflexes and the apnea test, brain death was determined within 48 hours and we lost our patient on 92nd day of ECMO.

\section{Discussion}

The conventional ventilatory support versus ECMO for severe adult respiratory failure(CESAR) trial in 2009 showed that ECMO therapy improved the patient outcome in terms of survival and a large number of patients benefited from ECMO therapy for respiratory failure (63 vs. $47 \%$ ). ${ }^{2}$ Though not positive in the traditional sense, the recently published ECMO to Rescue Lung Injury in Severe Acute Respiratory Distress Syndrome (EOLIA) trial showed favorable results in the group randomized to ECMO. ${ }^{4} \mathrm{~A}$ subsequent post hoc Bayesian analysis confirmed a high probability of benefit, as well as the encouraging safety profile seen in EOLIA and has resulted in a greater acceptance of ECMO by the critical care community in general. ${ }^{5} \mathrm{~A}$ subsequent meta-analysis of studies of ECMO for ARDS also confirmed a mortality benefit at 60 days. ${ }^{6}$ In addition, there have been case reports of successful ECMO therapy in the elderly population, with a survival rate of $41 \%{ }^{3,7}$ Further, studies have shown that prolonged ECMO is associated with a $45 \%$ hospital survival rate. ${ }^{8}$

As per ELSO guidelines, VV-ECMO should be instituted for respiratory failure in early phase of ARDS when lung damage is considered reversible and should be avoided in case of more than 7 days of mechanical ventilation, ${ }^{9-11}$ but it is difficult to follow this recommendation rigidly and therefore some deviations are often seen. The reason for this is multifactorial - the varied progression of ARDS pathology, late patient referral, late arrival in the tertiary care hospital and the socioeconomic obstacles. In ARDS, an exudative phase is followed by a proliferative stage, with resolution of edema and proliferation of alveolar cells and fibroblasts. This initial phase has a duration of $\sim 7$ to 10 days. In some patients, ARDS then resolves while in others progresses to a phase with fibrosing alveolitis. ${ }^{12,13}$ The best chance to treat ARDS therefore lies in the first week of mechanical ventilation before fibrotic lung changes occur. We in our patient could institute ECMO on 8th day of mechanical ventilation because of the situation of transfer from another hospital and finance arrangements, etc.

The number of complications, for example, infection, renal failure, and coagulopathy, associated with long ECMO run duration may prompt clinical decision to terminate the treatment after a prescribed period of time. However, this therapy may be extended if the primary cause of respiratory failure necessitating ECMO is expected to resolve and there have been cases where ECMO supports were continued for more than 2 weeks. ${ }^{14,15}$ Also if lung transplant facility is available and feasible, in case the lungs did not improve on ECMO 
therapy, then longer ECMO run can be considered, provided the other vital organ (brain, kidney, and liver) functions are preserved and the patient is well treated for the pulmonary infection.

Acute respiratory infection with a defined pathogen(s) as a sole cause of the respiratory failure can be a candidate for extended ECMO treatment. Data on successful outcomes in the 2009 H1N1 influenza A outbreak support the possibility of applying long-term ECMO treatment to acute respiratory failure caused by a superadded bacterial infection with an identifiable pathogen that can be treated with appropriate chemotherapy. ${ }^{16}$ The recommended treatment duration for culture positive bacterial sepsis ranges from 10 to 14 days, indicating a possibility for applying long-term ECMO. Our patient, in fact, could be treated successfully for her recurrent bacterial infections and sepsis by effective antimicrobials.

Complications such as coagulopathies (bleeding, thromboembolism, disseminated intravascular coagulation), membrane oxygenator failure, infection, liver dysfunction, and kidney failure may lead to shortening or termination of ECMO. We in our patient faced complications such as bleeding, oxygenator thrombosis, multiple sepsis episodes, thrombocytopenia, and renal impairment in episodes, but we could treat them effectively, which gave the hope of patient recovery so we continued the ECMO therapy. Unfortunately, the pulmonary parenchyma did not improve for quite a long period of almost 12 weeks of ECMO support and finally when the lungs showed signs of recovery, an acute neurological complication ensued that was irreversible and fatal.

Our patient had several episodes of intrapulmonary hemorrhages, hematuria, gastrointestinal hemorrhage needing large amount of blood and product transfusions, feeding interruptions, skin and musculoskeletal issues including ankle and knee stiffness. She required repositioning and fixing of the ECMO cannulae in position intermittently. Our experience showed that the ECMO support can be prolonged by successfully managing these problems with coordinated multidisciplinary approach and other supportive care such as tracheostomy, active mobilization on bed, physiotherapy, nutrition, and psychological support.

We thought about the possibility of lung transplantation as a salvage therapeutic option in our patient with end-stage lung disease. There were only few case reports in a select group of patients with severe ARDS ${ }^{17}$ that were bridged to lung transplantation. However, there was a lack of sufficient data on its use in patients with ARDS due to infection, tracheostomy, long mechanical ventilation, and multiple episodes of gram-negative sepsis, renal impairment, and morbid obesity. ${ }^{18}$ Prolonged ECMO can become futile due to irreversibility of lung pathology when lung transplantation is not an option as happened in our patient.

We encountered nonhealing of fibrotic changes of lung parenchyma due to severe form of ARDS, thus prolonging the ECMO run; similar experiences have been shared by many authors-Ali et al reported an ECMO run of 146 days for severe ARDS following pneumonia. ${ }^{14}$ Umei et al had their experience of 403 days long ECMO run for idiopathic pulmonary fibrosis while waiting for a lung transplant. ${ }^{15}$ Kim et al described a 107 days ECMO run for interstitial pneumonia as a bridge to lung transplant. ${ }^{19}$ Raza et al reported a 300 days ECMO run for severe fibrotic lung disease. ${ }^{20}$

We faced ECMO-related issues such as membrane failure and thrombosis requiring change of membrane oxygenator twice during this 92days course of ECMO. Similar issues were faced by Kim et $\mathrm{al}^{19}$ and Raza et $\mathrm{al}^{20}$ and both the authors reported changing their oxygenator up to four times. Umei et al changed the membrane oxygenator 23 times and the cannulae 10 times without complication. ${ }^{18}$ Ali et al had to change theirs seven times. ${ }^{17}$

We in our case did not encounter heparin-induced thrombocytopenia and thrombosis (HITT) or heparin resistance during the 3-month period of ECMO run. Raza et al reported HITT in their case requiring switching of heparin over to argatroban..$^{20}$

Extensive irreversible fibrotic lung changes in severe ARDS were the biggest reason for the prolonged ECMO run in our case, ECMO was instituted after 7 days of invasive ventilation, and probably fibrotic phase of ARDS had set in by then. Similar experiences were quoted by Ali et $\mathrm{al}^{17}$ who noted irreversible interstitial fibrosis and connective tissue changes in their patient.

Prolonged ECMO patient care with no recovery of lung pathology or no possibility of destination therapy ("ECMO to nowhere") can be extremely stressful for caregivers and the patient's family. Our patient was not a suitable candidate for lung transplantation. Ali et al and Raza et al also described similar difficulties in their study. ${ }^{17,20}$ But the authors Umei et al and Kim et al were able to bridge the ECMO to lung transplantation successfully in their patients. ${ }^{18,19}$

Adequate nutrition, mobilization, and regular on bed physiotherapy are extremely important to maintain musculoskeletal function. We continued chest, limb muscle, and joint physiotherapy for our patient. Active mobilization and physiotherapy are advocated by others too. ${ }^{19,20}$ Tracheostomy has been adopted by many authors ${ }^{18,19}$ for better pulmonary toileting and care while supporting the patients on longer ECMO run; we also performed tracheostomy in our patient on 12th ECMO day in view of long mechanical ventilation and for better pulmonary care.

In our patient, it was convulsions followed by irreversible apneic unconsciousness with absent brain stem reflexes that led to declaration of brain death and discontinuation of ECMO. Major neurological events have devastating outcomes in VV-ECMO patients and are not uncommon and brain death happens in around $23.5 \%$ of all neurological insults as described by Lurosso et $\mathrm{al}^{21}$ and determination of brain death is also another challenge in these patients on ECMO support as well described by Lie et al. ${ }^{22}$

\section{Conclusions}

We discussed a case of a 40-year-old woman with severe ARDS following H1N1 pneumonia with secondary bacterial sepsis, who was supported with VV-ECMO for intractable hypoxic respiratory failure. ECMO was initiated on 8th day of 
mechanical ventilation and was maintained for 92 days. We faced many challenges related to patient and machine factors and troubleshoot them successfully managing the longest run ECMO in our hospital but for the last major neurological insult leading to brain death in our patient. Our experience showed that ECMO can be used for a longer period than is conventionally recommended provided the primary cause of respiratory failure is expected to resolve with specific treatment or feasibility of lung transplantation is an available option in case of nonrecovery of lung pathology. Among many lessons we learned, most important is the decision to institute ECMO in time before irreversible pathological lung changes set in, not only to develop a good team dynamics to troubleshoot the machine issues but also to handle the complications in timely manner and to learn to prevent neurological insults including brain death. Not the least frequent debriefing sessions with the patient relatives, psychological support to the patient, and ethical consultations are extremely important during care of these patients on long ECMO run.

\section{Fundings}

Nil.

\section{Competing Interests}

The authors declare that they have no competing interests.

\section{Conflict of Interest}

None declared.

\section{References}

1 ELSO guidelines. Extracorporeal Life Support Organization (ELSO) Guidelines for Adult Respiratory Failure; Ver 1.4: August, 2017. Available at: https://www.elso.org/Portals/0/ ELSO\%20Guidelines\%20For\%20Adult\%20Respiratory\%20 Failure\%201_4.pdf. Accessed March 21, 2020

2 Peek GJ, Mugford M, Tiruvoipati R, et al; CESAR trial collaboration. Efficacy and economic assessment of conventional ventilatory support versus extracorporeal membrane oxygenation for severe adult respiratory failure (CESAR): a multicentre randomised controlled trial. Lancet 2009;374(9698) :1351-1363

3 Mendiratta P, Tang X, Collins RT II, Rycus P, Brogan TV, Prodhan P. Extracorporeal membrane oxygenation for respiratory failure in the elderly: a review of the Extracorporeal Life Support Organization registry. ASAIO J 2014;60(4):385-390

4 Combes A, Hajage D, Capellier G, et al; EOLIA Trial Group, REVA, and ECMONet. Extracorporeal membrane oxygenation for severe acute respiratory distress syndrome. N Engl J Med 2018;378(21):1965-1975

5 Goligher EC, Tomlinson G, Hajage D, et al. Extracorporeal membrane oxygenation for severe acute respiratory distress syndrome and posterior probability of mortality benefit in a Post Hoc Bayesian analysis of a randomized clinical trial. JAMA 2018;320(21):2251-2259
6 Munshi L, Walkey A, Goligher E, Pham T, Uleryk EM, Fan E. Venovenous extracorporeal membrane oxygenation for acute respiratory distress syndrome: a systematic review and meta-analysis. Lancet Respir Med 2019;7(2):163-172

7 Zapol WM, Snider MT, Hill JD, et al. Extracorporeal membrane oxygenation in severe acute respiratory failure. A randomized prospective study. JAMA 1979;242(20):2193-2196

8 Posluszny J, Rycus PT, Bartlett RH, et al; ELSO Member Centers. Outcome of adult respiratory failure patients receiving prolonged (14 Days) ECMO. Ann Surg 2016;263(3):573-581

9 Noah MA, Peek GJ, Finney SJ, et al. Referral to an extracorporeal membrane oxygenation center and mortality among patients with severe 2009 influenza $A(H 1 N 1)$ JAMA 2011;306(15):1659-1668

10 Peek GJ, Moore HM, Moore N, Sosnowski AW, Firmin RK. Extracorporeal membrane oxygenation for adult respiratory failure. Chest 1997;112(3):759-764

11 Kolla S, Awad SS, Rich PB, Schreiner RJ, Hirschl RB, Bartlett RH. Extracorporeal life support for 100 adult patients with severe respiratory failure. Ann Surg 1997;226(4):544-564, discussion 565-566

12 Pierrakos C, Karanikolas M, Scolletta S, Karamouzos V, Velissaris D. Acute respiratory distress syndrome: pathophysiology and therapeutic options. J Clin Med Res 2012;4(1):7-16

13 Ware LB, Matthay MA. The acute respiratory distress syndrome. N Engl J Med 2000;342(18):1334-1349

14 Ali AS, Yosri M, Abouelwafa M, et al. One hundred forty six days on ECMO: our longest ECMO run. Egypt J Crit Care Med 2018;6:113-121

15 Umei N, Ichiba S, Sakamoto A. Idiopathic pulmonary fibrosis patient supported with extracorporeal membrane oxygenation for 403 days while waiting for a lung transplant: a case report. Respir Med Case Rep 2018;24:86-88

16 Davies A, Jones D, Bailey M, et al; Australia and New Zealand Extracorporeal Membrane Oxygenation (ANZ ECMO) Influenza Investigators. Extracorporeal membrane oxygenation for 2009 Influenza $\mathrm{A}(\mathrm{H} 1 \mathrm{~N} 1)$ acute respiratory distress syndrome. JAMA 2009;302(17):1888-1895

17 Chang Y, Lee SO, Shim TS, et al. Lung transplantation as a therapeutic option in acute respiratory distress syndrome. Transplantation 2018;102(5):829-837

18 Weill D. Lung transplantation: indications and contraindications. J Thorac Dis 2018;10(7):4574-4587

19 Kim EJ, Paik HC, Park MS, et al. One hundred and seven days of ECMO as a bridge to lung transplantation: the longest duration among elderly patients. Korean. J Crit Care Med 2014;29(1):48-51

20 Raza T. 300 plus days on ECMO - Lessons Learned. Qatar Medical Journal, 4th Annual ELSO-SWAC Conference Proceedings 2017: 49

21 Lorusso R, Gelsomino S, Parise O, et al. Neurological injury in adults supported with venovenous extracorporeal membrane oxygenation for respiratory failure: findings from Extracorporeal Life Support Organization Database. Crit Care Med 2017;45(8):1389-1397

22 Lie SA, Hwang NC. Challenges of brain death and apnea testing in adult patients on extracorporeal membrane oxygenation-a review. J Cardiothorac Vasc Anesth 2019;33(8):2266-2272 